\title{
Effectiveness of Long-Term Physiotherapy in Parkinson's Disease: A Systematic Review and Meta-Analysis
}

\author{
Yohei Okada $^{\mathrm{a}, *}$, Hiroyuki Ohtsuka ${ }^{\mathrm{b}}$, Noriyuki Kamata ${ }^{\mathrm{c}}$, Satoshi Yamamoto ${ }^{\mathrm{d}}$, Makoto Sawada ${ }^{\mathrm{e}}$, \\ Junji Nakamura $^{\text {a,f }}$, Masayuki Okamoto ${ }^{\mathrm{g}}$, Masaru Narita ${ }^{\mathrm{a}, \mathrm{h}}$, Yasutaka Nikaido ${ }^{\mathrm{i}}$, Hideyuki Urakami ${ }^{\mathrm{i}}$, \\ Tsubasa Kawasaki ${ }^{\mathrm{j}}$, Shu Morioka ${ }^{\mathrm{a}}$, Koji Shomoto ${ }^{\mathrm{a}}$ and Nobutaka Hattori ${ }^{\mathrm{k}}$ \\ ${ }^{a}$ Graduate School of Health Sciences, Kio University, Nara, Japan \\ ${ }^{\mathrm{b}}$ Department of Physical Therapy, Showa University School of Nursing and Rehabilitation Sciences, \\ Kanagawa, Japan \\ ${ }^{\mathrm{c}}$ Department of Rehabilitation, Osaka University Hospital, Osaka, Japan \\ ${ }^{\mathrm{d}}$ Department of Physical Therapy, School of Health Sciences, Ibaraki Prefectural University of Health Sciences, \\ Ibaraki, Japan \\ e Department of Rehabilitation Medicine, National Hospital Organization Tottori Medical Center, Tottori, Japan \\ ${ }_{\mathrm{f}}^{\mathrm{f}}$ Department of Rehabilitation Medicine, Nishiyamato Rehabilitation Hospital, Nara, Japan \\ $\mathrm{g}^{\mathrm{g}}$ Department of Rehabilitation Medicine, Kakita Hospital, Kyoto, Japan \\ ${ }^{\mathrm{h}}$ Department of Rehabilitation, Hokuyukai Neurological Hospital, Hokkaido, Japan \\ ${ }^{\mathrm{i}}$ Department of Rehabilitation, Osaka Medical and Pharmaceutical University Hospital, Osaka, Japan \\ ${ }^{\mathrm{j}}$ Department of Physical Therapy, School of Health Sciences, Tokyo International University, Saitama, Japan \\ ${ }^{\mathrm{k}}$ Department of Neurology, Juntendo University Graduate School of Medicine, Tokyo, Japan
}

Accepted 20 July 2021

Pre-press 4 August 2021

\begin{abstract}
.
Background: Long-term physiotherapy is acknowledged to be crucial to manage motor symptoms for Parkinson's disease (PD) patients, but its effectiveness is not well understood.

Objective: This systematic review and meta-analysis aimed to assess the evidence regarding the effectiveness of long-term physiotherapy to improve motor symptoms and reduce antiparkinsonian medication dose in PD patients.

Methods: Pubmed, Cochrane, PEDro, and CINAHL were searched for randomized controlled trials before August 31, 2020 that investigated the effectiveness of physiotherapy for 6 months or longer on motor symptoms and levodopa-equivalent dose (LED) in PD patients with Hoehn and Yahr stage 1-3. We performed random effects meta-analyses for long-term physiotherapy versus no/control intervention and estimated standard mean differences with 95\% confidence intervals (CIs). Levels of evidence were rated by the Grading of Recommendation Assessment, Development and Evaluation approach.

Results: From 2,940 studies, 10 studies involving 663 PD patients were assessed. Long-term physiotherapy had favorable effects on motor symptoms in off medication state $[-0.65,95 \%$ CI -1.04 to $-0.26, p=0.001]$ and LED $[-0.49,95 \%$ CI -0.89
\end{abstract}

${ }^{*}$ Correspondence to: Dr. Yohei Okada, Graduate School of Health Sciences, Kio University, 4-2-2 Umami-naka, Koryo-cho,
Kitakatsuragi-gun, Nara 635-0832, Japan. Tel.: +81 74554 1601; E-mail: y.okada@kio.ac.jp. 
to $-0.09, p=0.02]$. Subgroup analyses demonstrated favorable effects on motor symptoms in off medication state by aerobic exercise $[-0.42,95 \% \mathrm{CI}-0.64$ to $-0.20, p<0.001]$ and LED by multidisciplinary rehabilitation of primarily physiotherapy $[-1.00,95 \% \mathrm{CI}-1.44$ to $-0.56, p<0.001]$. Quality of evidence for aerobic exercise and multidisciplinary rehabilitation were low and very low.

Conclusion: This review provided evidence that long-term physiotherapy has beneficial impact on motor symptoms and antiparkinsonian medication dose in PD patients and could motivate implementation of long-term physiotherapy.

Keywords: Physiotherapy, Parkinson's disease, motor symptoms, randomized controlled trial, systematic review, metaanalysis

\section{INTRODUCTION}

Parkinson's disease (PD) is a common progressive neurodegenerative disease characterized by various motor and non-motor symptoms from the early disease stage. As the disease progresses, these symptoms, along with impairment of the activities of daily living (ADL), become more pronounced [1-3]. Pharmacotherapy relieves these symptoms, but its effects become limited with the disease progression, requiring increases in medication dose [4, 5]. Increased medication, in turn, is likely to increase the risk of motor complications (such as motor fluctuations and dyskinesia) [6]. The combination of long-term physiotherapy and medication is widely acknowledged to be crucial for PD patients [7]. It would be desirable if long-term physiotherapy could manage motor symptoms and impaired ADL over the long term and beginning at the earliest possible disease phase, such that only small increases of antiparkinsonian medication are needed.

A previous systematic review and a meta-analysis showed that physiotherapy achieves short-term improvements in motor symptoms and ADL in PD patients but did not examine the effectiveness of longterm-physiotherapy on motor symptoms or ADL or antiparkinsonian medication dose [8,9]. Over the last decade, several randomized controlled trials (RCTs) have examined the effects of long-term physiotherapy of 6 months or longer on motor symptoms or ADL or antiparkinsonian medication dose in PD patients [10-19]. A narrative review stated physiotherapy interventions of at least 6 months are effective to achieve clinically meaningful improvement in motor symptoms, but this review was not systematic and did not include a meta-analysis of the effectiveness of long-term physiotherapy [20]. Long-term physiotherapy has not been sufficiently implemented, possibly partly because the body of evidence remains insufficient. Previous studies on long-term physiotherapy have adopted various types of physiotherapy interventions, such as aerobic exercise, strength exercise, multimodal exercise, or multidisciplinary rehabilitation mainly consisting of physiotherapy. However, it has not been examined which type of intervention is most beneficial for PD patients.

We aimed to analyze the evidence regarding the effectiveness of long-term physiotherapy in patients with mild-to-moderate PD through a systematic review and meta-analysis of RCTs that investigated the effectiveness of physiotherapy for more than 6 months on motor symptoms, ADL, and antiparkinsonian medication dose in PD patients with Hoehn and Yahr (HY) stage 3 or less. Based on the previous reviews and clinical guidelines, we defined physiotherapy as any intervention focused on the enhancement of muscle strength, aerobic capacity, balance, gait, and functional mobility by means of cueing, cognitive movement strategies, and/or physical exercises [8, 20-22]. Since motor symptoms are affected by antiparkinsonian medication, we assessed the effectiveness of physiotherapy on motor symptoms in patients in both on and off medication state. Subgroup analyses were also performed to examine which types of physiotherapy intervention were most effective. We also rated the quality of evidence by the Grading of Recommendation Assessment, Development and Evaluation (GRADE) approach [23].

\section{MATERIALS AND METHODS}

\section{Search strategy and selection criteria}

A comprehensive search was performed on September 1, 2020 to find RCTs published up to August 31, 2020 across the Pubmed, Cochrane Central Register of Controlled Trials, Physiotherapy Evidence (PEDro), and CINAHL databases. The search clauses are presented in the Supplementary Material. A manual search was also performed among previously published relevant reviews to retrieve articles not covered in the database search [7,20].

Inclusion criteria were: (1) parallel RCTs, (2) English-language articles, (3) for mild and moderate 
(HY 1-3) PD patients, (4) with interventions at least once a week for 6 months or longer, (5) comparing physiotherapy intervention with no intervention or control intervention, (6) assessing the effects on Unified Parkinson's Disease Rating Scale (UPDRS) or Movement Disorder Society-UPDRS (MDSUPDRS) motor score and motor and ADL score and levodopa-equivalent dose (LED) [24-26]. Exclusion criteria were: (1) for patients with parkinsonism other than PD, (2) providing non-physiotherapy interventions (e.g., dance, tai-chi, yoga, Pilates, Qigong, music, boxing, noninvasive stimulation), (3) medication state in assessment is not clear, and (4) abstract or conference proceedings.

\section{Data analysis}

After removing duplicates, 11 reviewers (YO, HO, NK, SY, MS, JN, MO, MN, YN, HU, and TK) worked in pairs to screen each study through the titles and abstracts, then reviewed the full text of potential studies and selected the relevant studies in pairs as well. All disagreements between reviewers were resolved by their consensus after discussion, or inclusion of a third reviewer (YO or HO). Data were extracted from the included studies by the first author and checked by other reviewers (HO, NK, SY, MS, JN, MO, MN, $\mathrm{YN}$, and HU). The extracted data included participants (number of subjects, HY stage), intervention protocol, outcome measures, and medication state in assessment.

The primary outcome was UPDRS or MDSUPDRS ((MDS-)UPDRS) motor score. Secondary outcomes were (MDS-)UPDR ADL score and LED. (MDS-)UPDR motor and ADL scores were global standard measures of motor symptoms and ADL in PD [24, 25]. LED is obtained from the daily antiparkinsonian medication and is practically useful summary of total daily antiparkinsonian medication dose [26].

If the mean change from baseline to post-intervention and standard deviation (SD) were not shown, the first author contacted each study's corresponding author. For data presented in the median and interquartile range, the median was considered the mean and the standard deviation was calculated considering that interquartile range $=1.35 \times \mathrm{SD}$ [27] When only the mean value and SD before and after the intervention were presented, the mean change was calculated from the difference between mean values, and the SD for the mean change was calculated as $[8,27]$

$$
\operatorname{var}_{\text {diff }}=\operatorname{var}_{\text {post }}-2 r \sqrt{\left(\text { var }_{\text {pre }} \text { var }_{\text {post }}\right)}
$$

where var diff is the variance of the change score, $v a r_{p r e}$ the variance of the baseline score, $v^{2} r_{\text {post }}$ the variance of the post-intervention score and $r$ the correlation between the baseline and post-intervention scores. The correlation co-efficient was assumed to be 0.5 to conservatively estimate the results $[8,27]$. For studies with 3 arms or more, the same control data were used for each comparison.

We conducted the meta-analyses to estimate the overall effects of long-term physiotherapy versus no/control intervention, following the design of previous meta-analyses [8, 9]. For each type of physiotherapy intervention employed in at least two studies, subgroup analyses were conducted to examine which type was most effective compared to no/control intervention. Standardized mean differences (SMD) with their 95\% confidence intervals (CIs) were calculated using a random effect model because of the possible clinical and methodological differences among trials. The test for heterogeneity was conducted using $\mathrm{I}^{2}$ statistics. All meta-analytical findings were calculated using Review Manager 5.4. The $\alpha$ level was set at 0.05 .

The methodological quality in each study was rated by 10 reviewers (YO, HO, NK, SY, MS, JN, MO, $\mathrm{MN}$, YN, and $\mathrm{HU}$ ) working in pairs. Risk of bias of the RCTs was assessed using the Cochrane Riskof-Bias tool. Domains of risk-of-bias assessments were (1) selection bias (random sequence generation, allocation concealment, and baseline comparability), (2) performance bias (blinding of participants and personnel, and cointerventions), (3) detection bias (blinding of outcome assessment), (4) attrition bias (intention to treat analysis and incomplete outcome data), (5) reporting bias (selective reporting), (6) other bias. The score of each bias domain was graded as low, high, or unclear.

Overall quality of evidence was assessed by GRADE approach by two independent reviewers (YO and HO). All studies were RCTs, so the evidence level started at high. The level of evidence was downgraded due to risk of bias, imprecision, inconsistency, indirectness, and publication bias, based on the GRADE guidelines [21]. Final quality of evidence was rated as high, moderate, low, or very low.

The protocol for this systematic review and metaanalysis was registered in the International Prospective Register of Systematic Reviews-PROSPERO (registry no. CRD42020206939) and is 
available online (https://www.crd.york.ac.uk/prospe ro/display_record.php?ID=CRD42020206939). This study was conducted according to the Cochrane group recommendations and reported according to the PRISMA (Preferred Reporting for Systematic Review and Meta-Analyses) guidelines [28].

\section{RESULTS}

\section{Study selection and characteristics}

The search identified 5,187 studies, of which 2,247 duplicates were removed. The remaining 2,940 studies were assessed for eligibility, and 2,820 studies were excluded by title and abstract screening. Eligibility of the remaining 120 studies was assessed by full-text review, resulting in a final 10 studies [10-19]. Figure 1 shows the reasons for exclusion. Study characteristics are summarized in Supplementary Table 1 . The number of subjects in each study was $66.3 \pm 41.7$ (mean \pm SD), with an overall total of 663 PD patients. Three studies were examined in each of 3 arms, so the control data were used twice [14-16]. Eligible studies investigated the effects of physiotherapy, such as aerobic exercise [14-16, 18, 19], resistance exercise [10,11], multimodal exercise [15, 17], or multidisciplinary rehabilitation mainly consisting of physiotherapy [12, 13]. The intervention duration ranged from 6 months to 2 years. Eight studies provided intervention of more than 6 months [10, 11, 14-19], 1 study provided intervention of 4 weeks followed by 1 year of learned-exercise maintenance [12], and 1 study provided intervention of 4 weeks at 1 year interval and maintenance of learned exercise for 2 years [13]. In 5 studies, the (MDS-)UPDRS motor score was evaluated only in on medication state [11, $12,14,15,17]$, in 3 studies it was examined only in off medication state $[13,16,18]$, and in 2 studies it was examined in both medication states [10,19].

\section{Risk of bias}

Figure 2 provides a summary of the bias risk, and Fig. 3 graphs the risk of bias across the 10 included studies. None of the 10 studies had a low risk of bias for all 11 items. All studies presented low bias risk for blinding of outcome assessment and other bias. Most of the studies had low risk of bias for random-sequence generation, allocation concealment, baseline comparability, cointerventions, and
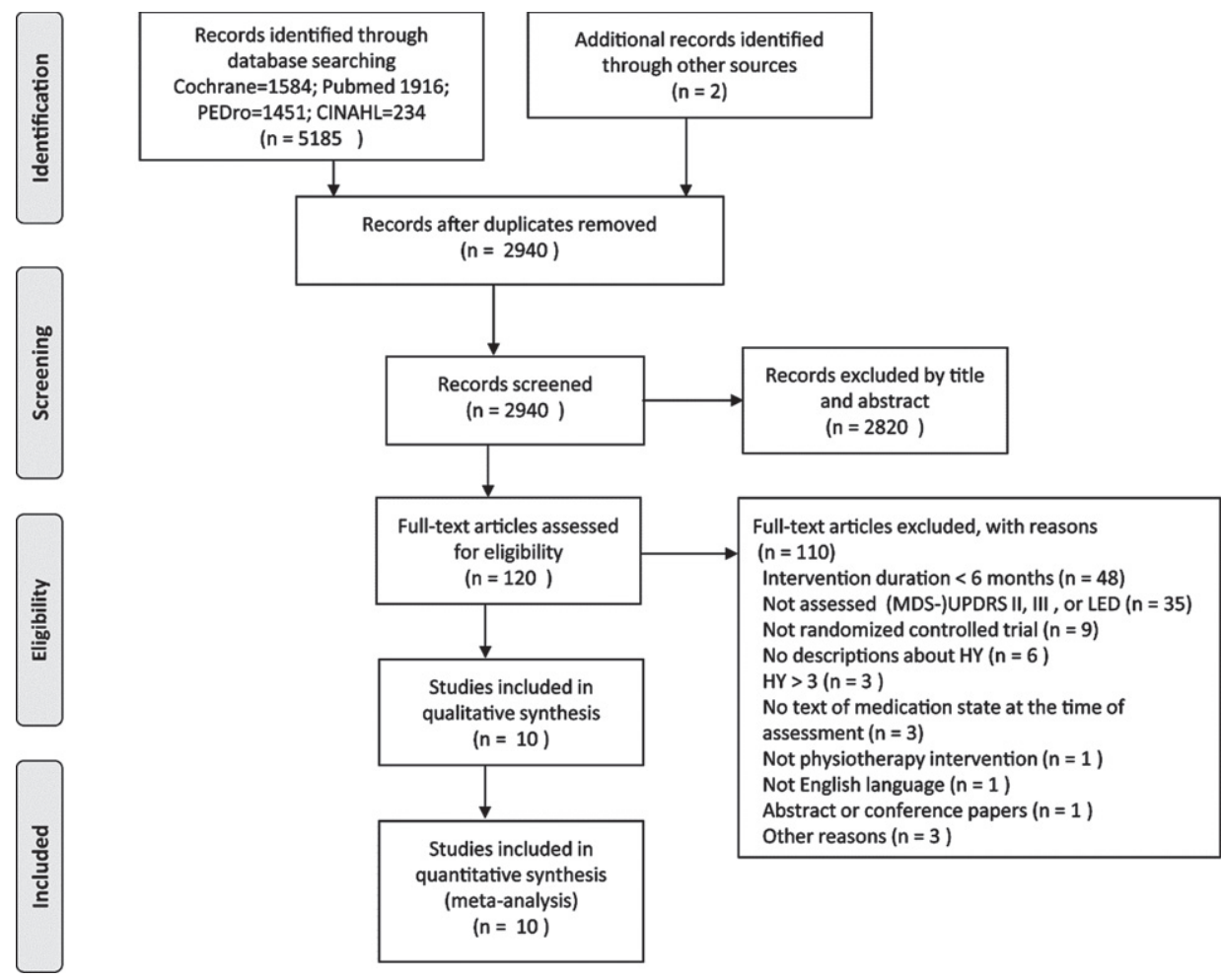

Fig. 1. Flow diagram of reviewing process based on the PRISMA statement. 
Table 1

Summary of findings

\begin{tabular}{|c|c|c|c|c|c|c|c|c|c|}
\hline & Outcome & $\begin{array}{c}\text { Number of } \\
\text { subjects } \\
\text { (studies) }\end{array}$ & $\begin{array}{c}\text { SMD } \\
{[95 \% \mathrm{CI}]}\end{array}$ & Risk of bias & Inconsistency & Imprecision & Indirectness & $\begin{array}{l}\text { Publication } \\
\text { bias }\end{array}$ & $\begin{array}{c}\text { Levels } \\
\text { of evidence } \\
\text { (GRADE) }\end{array}$ \\
\hline \multirow{7}{*}{$\begin{array}{l}\text { Aerobic } \\
\text { exercise }\end{array}$} & (MDS-)UPDRS & $\begin{array}{c}245 \\
(4 \text { RCTs) }\end{array}$ & $\begin{array}{c}-0.17 \\
-0.42018\end{array}$ & $\begin{array}{l}\text { Downgraded } \\
\text { by one level }\end{array}$ & $\begin{array}{c}\text { No } \\
\text { downorading }\end{array}$ & $\begin{array}{c}\text { No } \\
\text { downorading }\end{array}$ & $\begin{array}{c}\text { No } \\
\text { downorading }\end{array}$ & $\begin{array}{l}\text { Downgraded } \\
\text { by one leveld }\end{array}$ & $(+)(+)()()$ \\
\hline & (MDS-)UPDRS & 330 & -0.40 & Downgraded & No & No & No & Downgraded & $(+)(+)()()$ \\
\hline & motor off & (4 RCTs) & {$[-0.64,-0.20]$} & by one level & downgrading & downgrading & downgrading & by one level ${ }^{\mathrm{d}}$ & Low \\
\hline & (MDS-)UPDRS & 295 & 0.08 & Downgraded & No & No & No & Downgraded & $(+)(+)()()$ \\
\hline & ADL & (5 RCTs) & {$[-0.14,0.31]$} & by one level & downgrading & downgrading & downgrading & by one level ${ }^{\mathrm{d}}$ & Low \\
\hline & LED & 125 & -0.18 & Downgraded & NA & No & No & Downgraded & $(+)(+)()()$ \\
\hline & & (1 RCT) & {$[-0.53,0.17]$} & by one level & & downgrading & downgrading & by one level ${ }^{\mathrm{d}}$ & Low \\
\hline \multirow{8}{*}{$\begin{array}{r}\text { Resistance } \\
\text { exercise }\end{array}$} & (MDS-)UPDRS & 73 & -0.30 & Downgraded & No & Downgraded & No & Downgraded & $(+)()()()$ \\
\hline & motor on & (2RCTs) & {$[-0.77,0.16]$} & by one level & downgrading & by one level ${ }^{\mathrm{a}}$ & downgrading & by one level ${ }^{d}$ & Very low \\
\hline & (MDS-)UPDRS & 38 & -0.90 & Downgraded & NA & Downgraded & No & Downgraded & $(+)()()()$ \\
\hline & motor off & (1RCT) & {$[-1.56,-0.22]$} & by one level & & by two level ${ }^{\mathrm{a}, \mathrm{b}}$ & downgrading & by one level ${ }^{\mathrm{d}}$ & Very low \\
\hline & (MDS-)UPDRS & 35 & 0.17 & Downgraded & NA & Downgraded & No & Downgraded & $(+)()()()$ \\
\hline & $\mathrm{ADL}$ & (1 RCT) & {$[-0.50,0.83]$} & by one level & & by two level ${ }^{\mathrm{a}, \mathrm{b}}$ & downgrading & by one level ${ }^{\mathrm{d}}$ & Very low \\
\hline & LED & 40 & -0.30 & Downgraded & NA & Downgraded & No & Downgraded & $(+)()()()$ \\
\hline & & (1 RCT) & {$[-0.92,0.33]$} & by one level & & by two level ${ }^{a, b}$ & downgrading & by one level ${ }^{\mathrm{d}}$ & Very low \\
\hline \multirow{6}{*}{$\begin{array}{l}\text { Multimodal } \\
\text { exercise }\end{array}$} & (MDS-)UPDRS & 80 & 0.11 & Downgraded & NA & Downgraded & No & Downgraded & $(+)()()()$ \\
\hline & motor on & (1 RCT) & {$[-0.33,0.55]$} & by one level & & by one level ${ }^{\mathrm{a}}$ & downgrading & by one level ${ }^{\mathrm{d}}$ & Very low \\
\hline & (MDS-)UPDRS & 108 & -1.26 & Downgraded & Downgraded & Downgraded & No & Downgraded & $(+)()()()$ \\
\hline & $\mathrm{ADL}$ & (2 RCTs) & {$[-3.11,0.60]$} & by one level & $\begin{array}{c}\text { by two level } \\
\left(\mathrm{I}^{2}=92 \%\right)\end{array}$ & by one level ${ }^{\mathrm{b}}$ & downgrading & by one level $^{\mathrm{d}}$ & Very low \\
\hline & LED & 39 & -0.08 & Downgraded & NA & Downgraded & No & Downgraded & $(+)()()()$ \\
\hline & & (1 RCT) & {$[-0.70,0.55]$} & by one level & & by one level ${ }^{\mathrm{a}}$ & downgrading & by one level ${ }^{\mathrm{d}}$ & Very low \\
\hline \multirow{7}{*}{$\begin{array}{l}\text { Multidisciplinary } \\
\text { rehabilitation }\end{array}$} & (MDS-)UPDRS & 50 & -1.35 & Downgraded & NA & Downgraded & Downgraded & Downgraded & $(+)()()()$ \\
\hline & motor on & (1 RCT) & {$[-1.97,-0.73]$} & by one level & & by two level ${ }^{a, b}$ & by one level ${ }^{c}$ & by one level ${ }^{\mathrm{d}}$ & Very low \\
\hline & (MDS-)UPDRS & 40 & $\begin{array}{r}-1.90 \\
-266-1\end{array}$ & Downgraded & NA & Downgraded & Downgraded & Downgraded & $\begin{array}{l}(+)()()() \\
\text { Verv low }\end{array}$ \\
\hline & (MDS-)UPDRS & $\begin{array}{c}(1 \mathrm{KL}) \\
90\end{array}$ & $\begin{array}{c}{[-2.06,-1.4} \\
-0.64\end{array}$ & Downgraded & No & Downgraded & Downgraded & Downgraded & $\begin{array}{l}\text { very low } \\
(+)()(O)\end{array}$ \\
\hline & ADL & (2RCTs) & {$[-1.06,-0.21]$} & by one level & downgrading & by one level ${ }^{\mathrm{a}}$ & by one level ${ }^{\mathrm{c}}$ & by one level ${ }^{\mathrm{d}}$ & Very low \\
\hline & LED & 90 & -1.00 & Downgraded & No & Downgraded & Downgraded & Downgraded & $(+)()()()$ \\
\hline & & (2RCTs) & {$[-1.44,-0.56]$} & by one level & downgrading & by one level ${ }^{\mathrm{a}}$ & by one level ${ }^{\mathrm{c}}$ & by one level ${ }^{\mathrm{d}}$ & Very low \\
\hline
\end{tabular}

(MDS-)UPDRS, (Movement Society-sponsored revision of) the Unified Parkinson's Disease Rating Scale; on, on medication state; off, off medication state; ADL, activities of daily living; LED, levodopa equivalent dose; RCT, randomized controlled trial; NA, not applicable; GRADE, Grading of Recommendation Assessment, Development and Evaluation. Imprecision: $a=$ total sample size $(<100), b=$ wide confidence intervals $(>1)$. Indirectness: $c=$ multidisciplinary rehabilitation mainly consisting of physiotherapy, but also including occupational therapy. Publication bias: $d=$ small number of studies. GRADE Working Group grades of evidence. High: We are very confident that the true effect is similar to the estimated effect. Moderate: We are moderately confident that the true effect is close to the estimated effect. Low: Our confidence in the effect estimate is limited; the true effect may be substantially different from the estimated effect. Very low: We have very little confidence in the effect estimate; the true effect is likely to be substantially different from the estimated effect. 


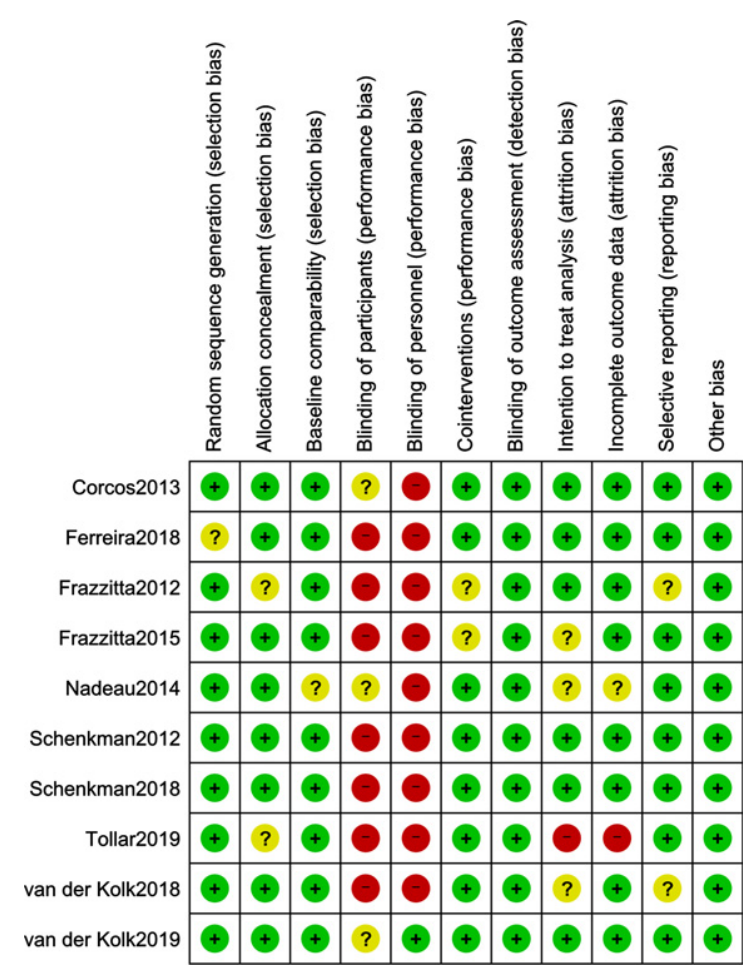

Fig. 2. Risk of bias summary: Review authors' judgements about each risk of bias item for each included study. Risk of bias for each domain in each study is represented in green for low risk of bias, red for high risk of bias, and yellow for unclear risk of bias.

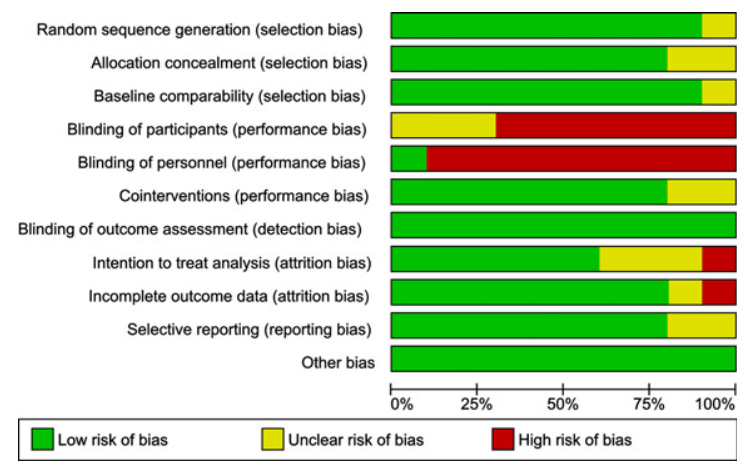

Fig. 3. Risk of bias graph: review authors' judgements about each risk of bias item presented as percentages across all included studies.

selective reporting, except for one or two studies that had unclear levels of bias for one or more of these items. For blinding of participants, the majority of studies had high risk of bias, and for blinding of personnel, all studies but one [19] had high risk.

\section{Effectiveness of long-term physiotherapy}

Six of 10 RCTs involving 448 subjects were included in the meta-analysis examining the effects on (MDS-)UPDRS motor score in on medication state (Fig. 4) [10-12, 14, 15, 19]. Two of the 6 studies were three-arm RCTs examining the effects of two different protocols: aerobic exercise or aerobic exercise plus multimodal exercise $[14,15]$. Pooled data showed a positive but not significant trend for physiotherapy $(p=0.06)$. Significant heterogeneity was observed between trials. Subgroup analysis revealed no significant effects for aerobic exercise $(n=4)$ or resistance exercise $(n=2)$. One study on multimodal exercise also showed no positive results [15]. Only one study of multidisciplinary rehabilitation mainly consisting of physiotherapy showed positive effects [12].

Five of 10 RCTs involving 408 subjects were included in the meta-analysis to examine the effects on (MDS-)UPDRS motor score in off medication state (Fig. 5) [10, 13, 16, 18, 19]. One study consisted of three-arm RCTs to test the effects of two different protocols of aerobic exercise [16]. Pooled data showed a significant effect in favor of physiotherapy (SMD $-0.65,95 \%$ CI -1.04 to -0.26 ). Evidence indicated significant heterogeneity between trials. Subgroup analysis showed significant positive effects of aerobic exercise (SMD - $0.42,95 \%$ CI -0.64 to -0.20 ). One study each on resistance exercise and multidisciplinary rehabilitation showed positive results $[10,13]$.

Seven of 10 RCTs involving 528 subjects were included in the meta-analysis to examine the effects on (MDS-)UPDRS ADL score (Fig. 6) [11-17]. Three of 7 trials examined the effects of two different protocols, aerobic exercise, or aerobic exercise plus multimodal exercise, hence both comparisons in these trials were included in the analysis [14-16]. Pooled data indicated no significant overall effect of physiotherapy. Significant heterogeneity was observed between trials. Subgroup analysis revealed no significant effects by aerobic exercise $(n=3$, $p=0.47)$ and multimodal exercise $(n=2, p=0.19)$, but a significant positive effect by multidisciplinary rehabilitation (SMD $-0.64,95 \% \mathrm{CI}-1.06$ to -0.21 ). One study on resistance exercise showed no positive effect [11].

Five of 10 RCTs involving 294 subjects were included in the meta-analysis to examine the effects on LED (Fig. 7) $[10,12,13,17,19]$. Pooled data showed significant effect in favor of physiotherapy ( $\mathrm{SMD}=-0.49,95 \% \mathrm{CI}-0.89$ to -0.09 ). Evidence indicated significant heterogeneity between trials. Subgroup analysis revealed significant positive effects of multidisciplinary rehabilitation (SMD 
(A)

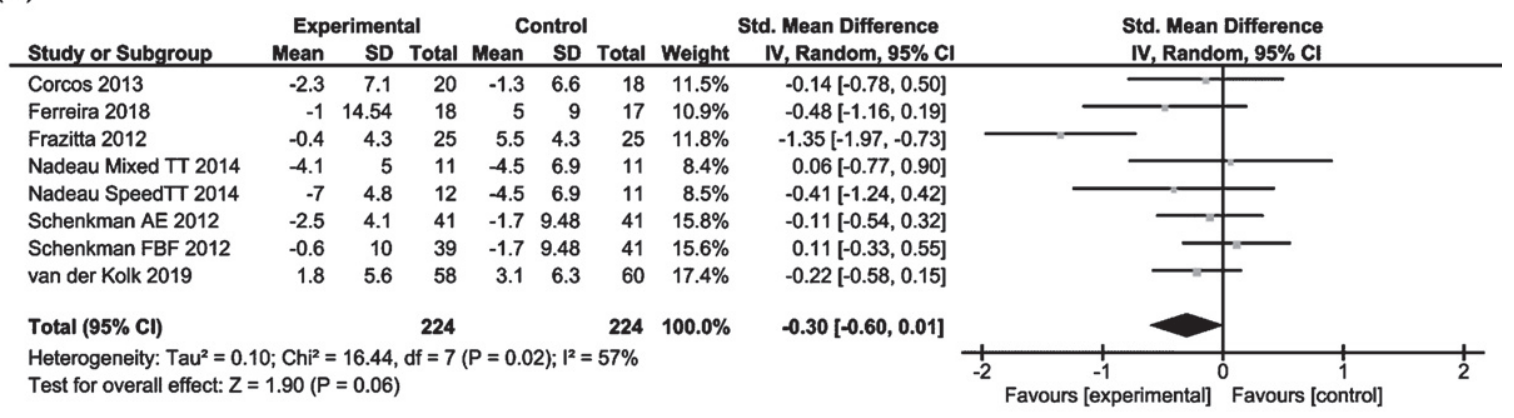

(B)

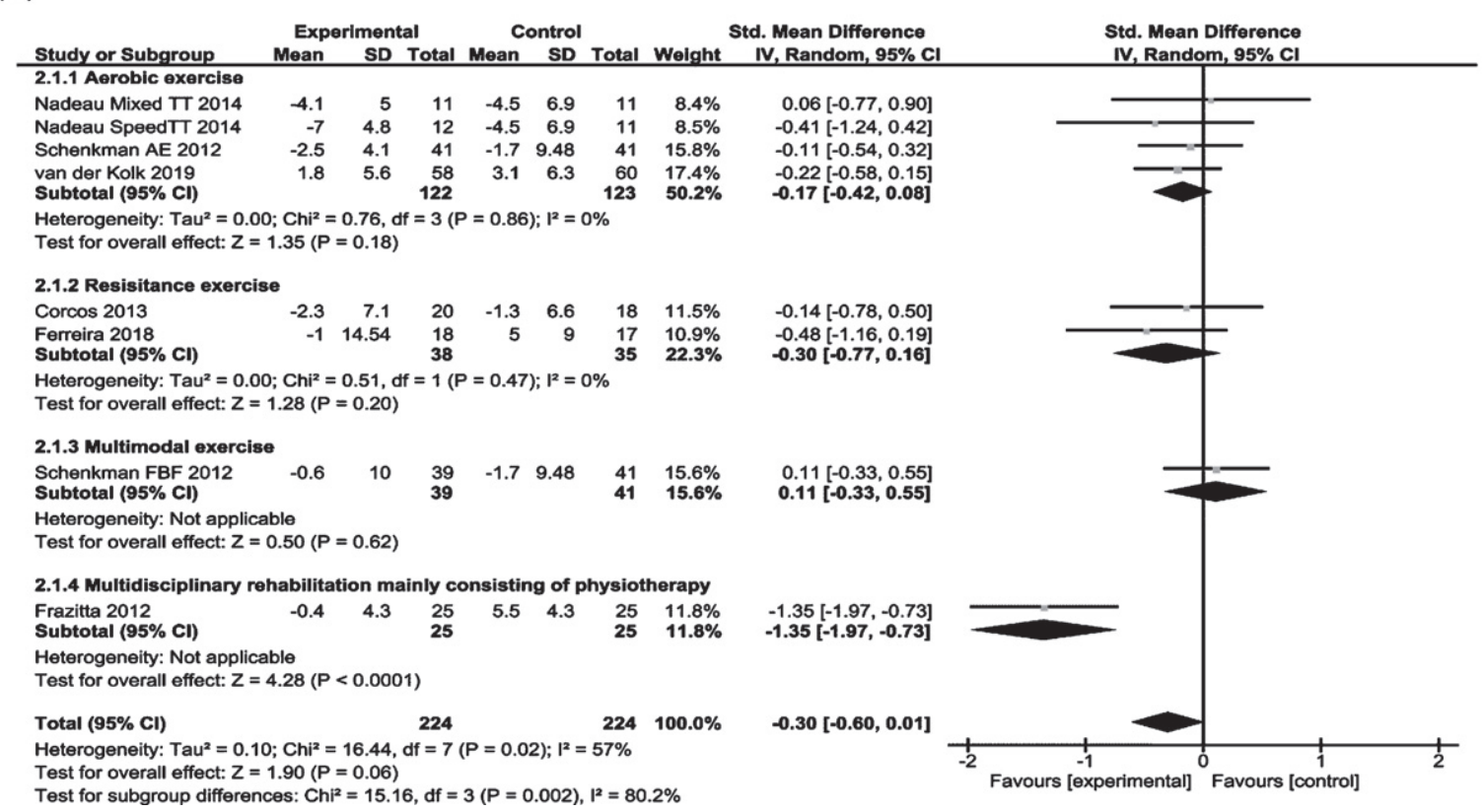

Fig. 4. Forest plots of MDS-UPDRS/UPDRS motor score in on medication state for physiotherapy versus no/control intervention. (A) Overall effect of physiotherapy interventions. (B) Subgroup analysis (category of intervention).

$-1.00,95 \% \mathrm{CI}-1.44$ to -0.56$)$. One study each on aerobic exercise, resistance exercise and multimodal exercise showed no positive results $[10,17,19]$.

\section{Quality of evidence}

The GRADE levels, and degree of downgrade for each element were detailed in Table 1. For most outcomes of interventions, the number of studies was so limited that it was impossible to perform statistical analyses of the funnel plots. Aerobic exercise for all outcomes was scored as "low". Resistance exercise, Multimodal exercise, and multidisciplinary rehabilitation for all outcomes were scored as "very low".

\section{DISCUSSION}

This is the first systematic review and meta-analysis to analyze the evidence regarding the effectiveness of long-term physiotherapy on motor symptoms, $\mathrm{ADL}$, and antiparkinsonian medication dose. We analyzed the overall effect of physiotherapy for each outcome and performed subgroup analysis to determine the most beneficial type of physiotherapy intervention. Our results provided evidence that long-term-physiotherapy for 6 months or longer for mild-to-moderate PD patients is effective for reducing motor symptoms in off medication state and for reducing the antiparkinsonian medication dose, compared to no/control intervention. Previous narrative reviews have highlighted the need for long-term 
(A)

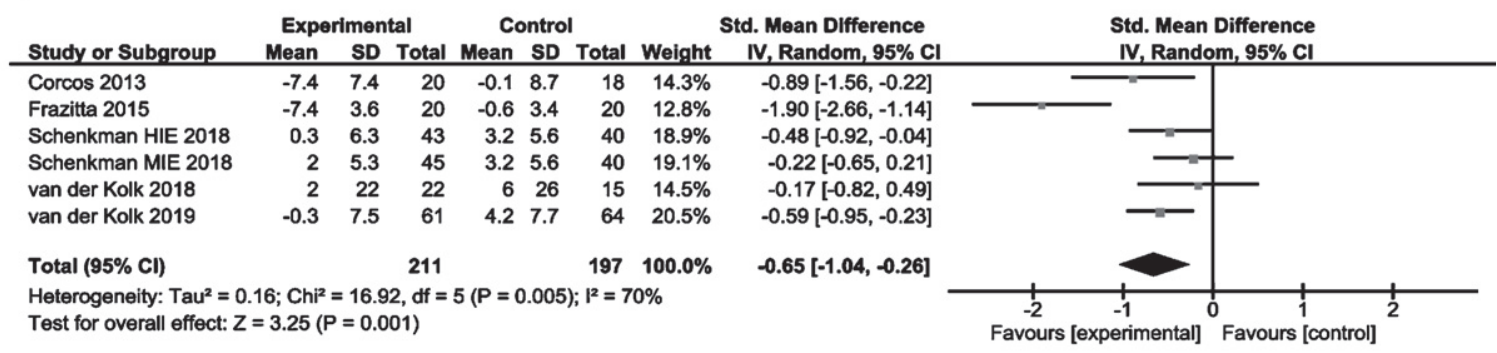

(B)

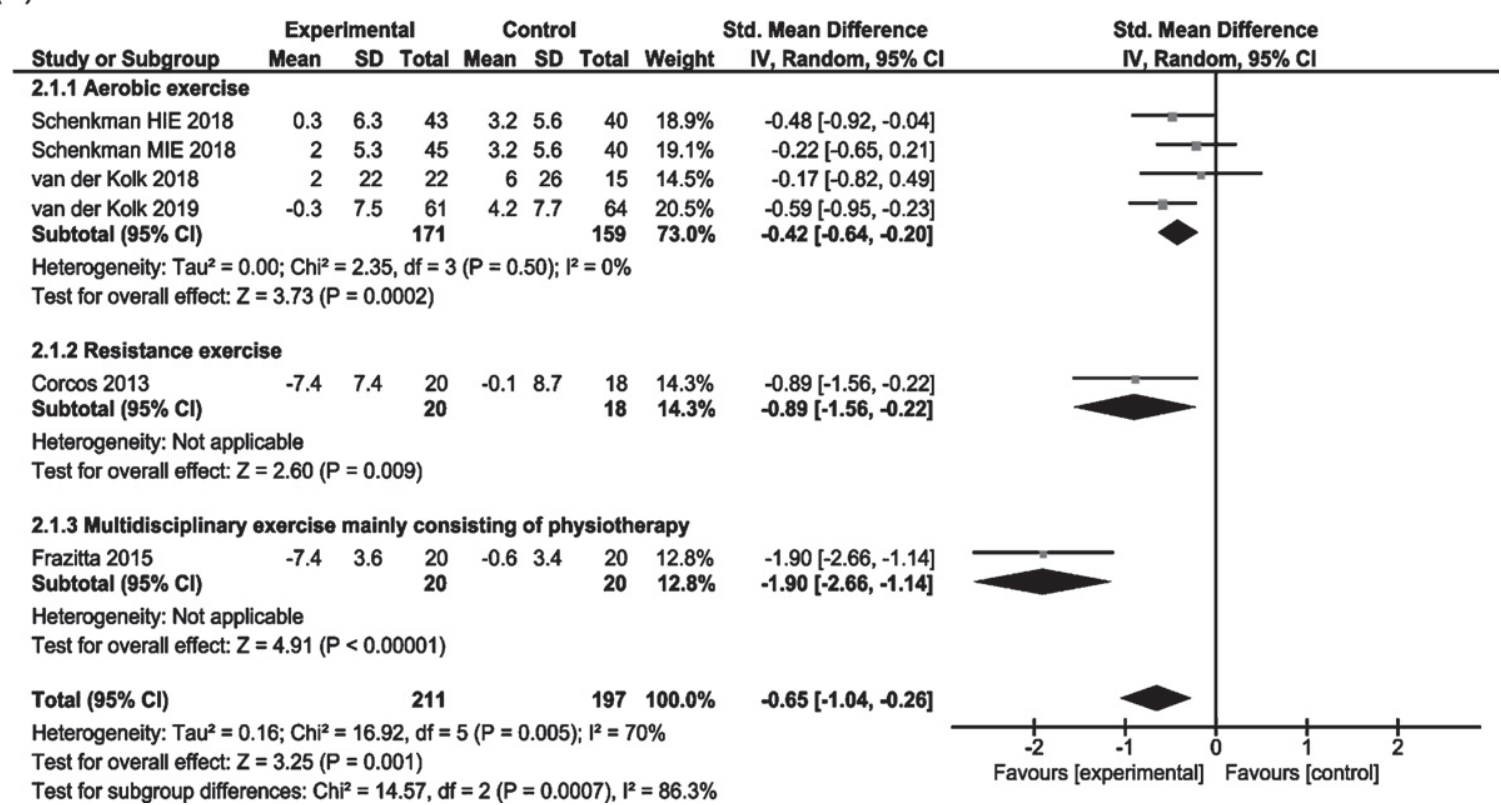

Fig. 5. Forest plots of MDS-UPDRS/UPDRS motor score in off medication state for physiotherapy versus no/control intervention. (A) Overall effect of physiotherapy interventions. (B) Subgroup analysis (category of intervention).

physiotherapy from the early phase of disease, and our results supported this notion [7, 20]. And, although the number of studies and subjects were small in our subgroup analyses, aerobic exercise showed favorable effects on motor symptoms in off medication state, and multidisciplinary rehabilitation mainly consisting of physiotherapy showed favorable effects on ADL and LED.

A strength of this review is that only studies in which the disease severity was clearly known to be HY 1-3 were selected for inclusion. This makes it possible to apply the evidence on long-term physiotherapy from this study for mild-to-moderate PD patients. The second strength is that we evaluated the effects of long-term physiotherapy for motor symptoms in both on medication and off medication state, separately. Since the antiparkinsonian medication state may affect the results, especially in regard to the (MDS-)UPDRS motor score, we strictly selected only those studies for which the medication state in assessment was known. Another strength is that we rated the quality of evidence in each study using a GRADE approach by two independent reviewers. This made it possible to provide objective information regarding the level of certainty for each result, which would be useful for applying the evidence to clinical practice.

Analysis of the overall effects of long-term physiotherapy on motor symptoms in off medication state was conducted in 5 studies, and favorable effects of long-term physiotherapy were observed $[10,13,16$, $18,19]$. Subgroup analysis revealed favorable effects of aerobic exercise. All these studies employed pure aerobic exercise at a rate of 3 or 4 times a week for 6 months, with consistent results observed [16, 18, 19]. A previous review showing the effects of 
(A)

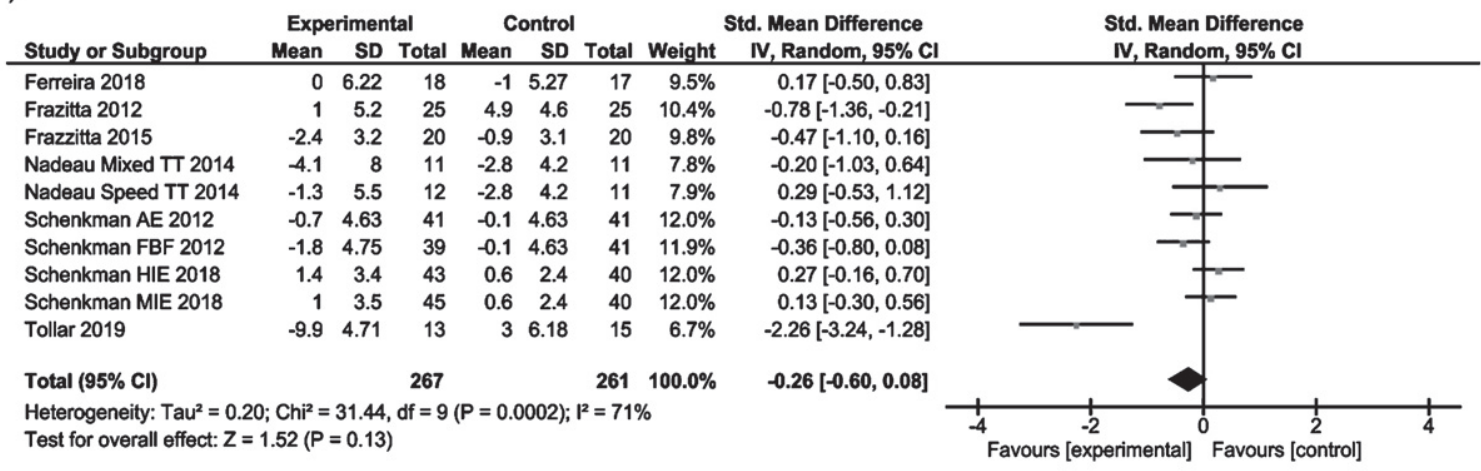

(B)

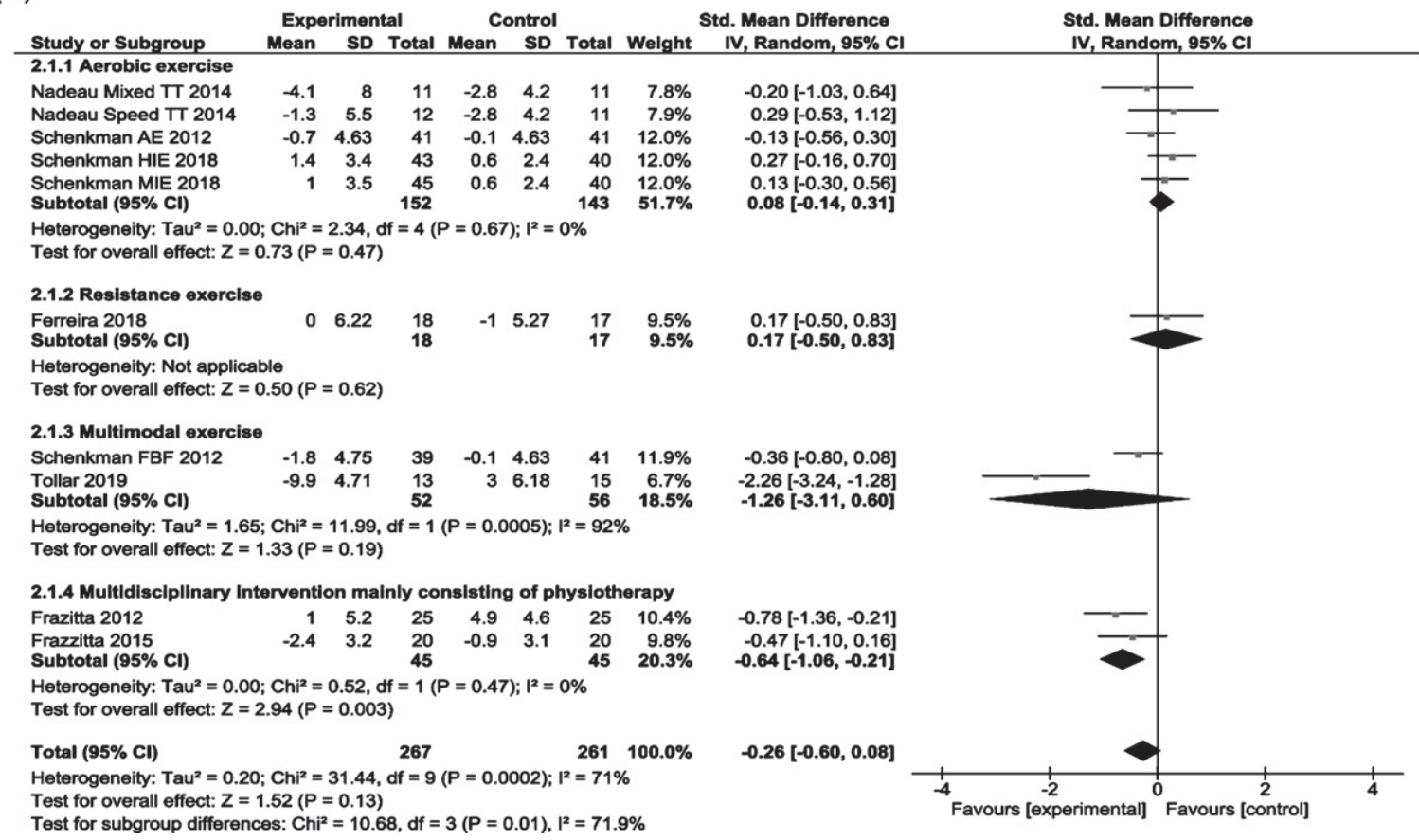

Fig. 6. Forest plots of MDS-UPDRS/UPDRS ADL score for physiotherapy versus no/control intervention. (A) Overall effect of physiotherapy interventions. (B) Subgroup analysis (category of intervention).

aerobic exercise on motor symptoms in off medication state included studies with interventions of at least 4 weeks [29]. In the present review, favorable effects were also observed with longer-term intervention of 6 months. Long-term aerobic exercise could have a unique effect on reducing motor symptoms in off medication state in mild-to-moderate PD patients. Moreover, although motor symptoms would be expected to worsen over a 6-month period, favorable effects of long-term aerobic exercise on motor symptoms were observed; this may have been the result of neuroplastic changes resulting in compensatory strategies for motor symptoms in PD patients [30].
The analysis of the overall effects of long-term physiotherapy on motor symptoms in on medication state included 6 studies $[10-12,14,15,19]$ and showed that long-term physiotherapy tended to improve motor symptoms, but not to a statistically significant difference. Since the effects of antiparkinsonian medications may be stronger than the effects of physiotherapy in on medication state, it may be difficult to observe the effect of long-term physiotherapy. One small study with no blinding of multidisciplinary rehabilitation mainly consisting of physiotherapy with a longer intervention period of 1 year showed huge effects on motor symptoms in on medication state [12], but other larger studies [15, 19] 
(A)

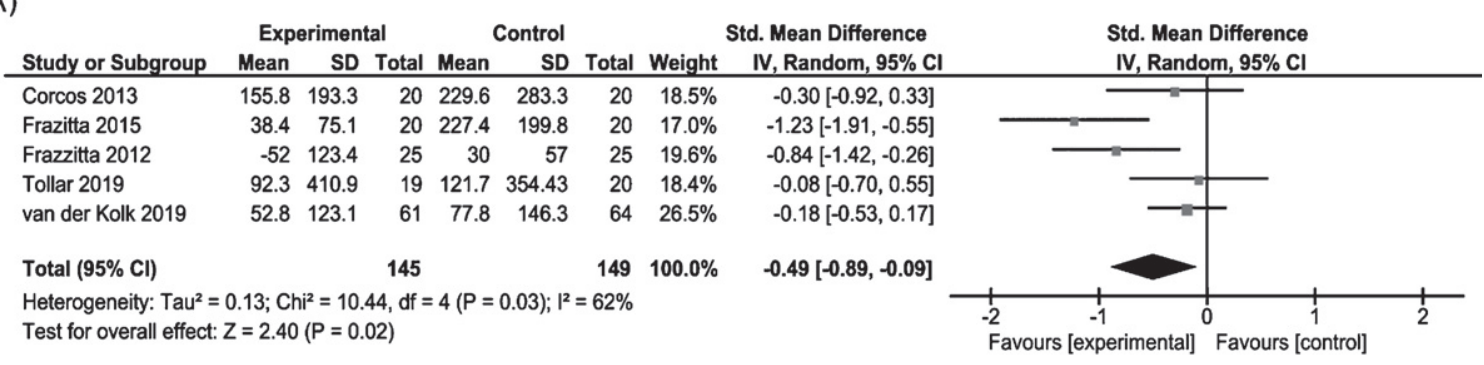

(B)

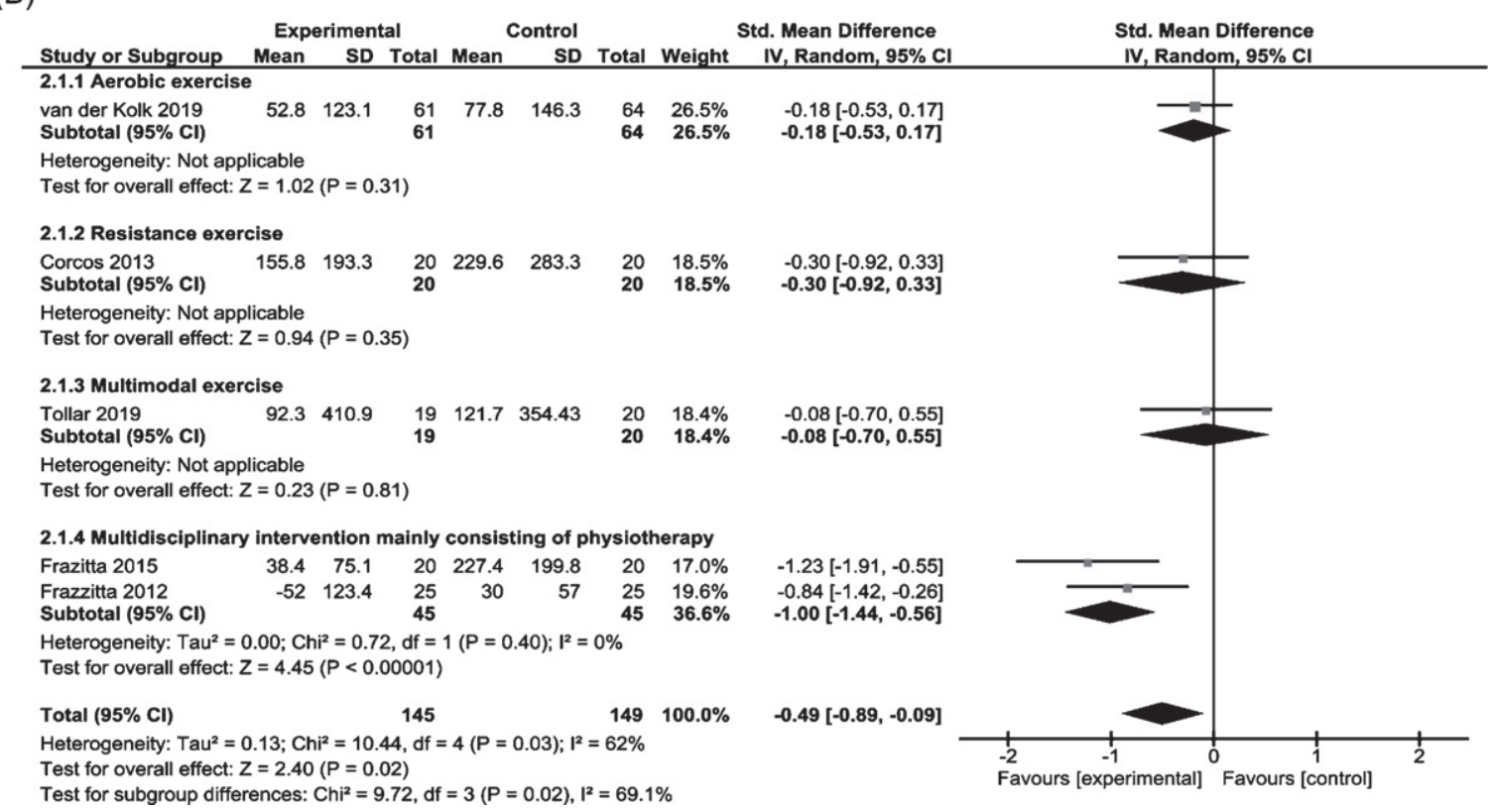

Fig. 7. Forest plots of Levodopa equivalent dose for physiotherapy versus no/control intervention. (A) Overall effect of physiotherapy interventions. (B) Subgroup analysis (category of intervention).

showed only small non-significant effects. The effects of multidisciplinary rehabilitation mainly consisting of physiotherapy on motor symptoms in on medication state may be more visible over a longer period of time, but the results of the small study should be considered with caution.

Our present analysis of the effects of long-term physiotherapy on ADL included 7 studies [11-17] and revealed no significant effect. Subgroup analysis by intervention category was performed and revealed favorable effects only from multidisciplinary rehabilitation. The two studies on multidisciplinary rehabilitation included in the subgroup analysis focused on physiotherapy, but also included occupational therapy $[12,13]$. A large RCT showed that occupational therapy can improve performance of ADL [31], and it could be considered that physiotherapy along with occupational therapy has a beneficial impact on ADL.
The analysis of the overall effects of long-term physiotherapy on LED included 5 studies $[10,12$, 13, 17, 19]. Favorable effects with heterogeneity were observed. Subgroup analysis revealed that a favorable effect was observed only from multidisciplinary interventions. Long-term multidisciplinary rehabilitation was also found to contribute to a reduction in antiparkinsonian medication dose. The two studies included in the subgroup analysis on multidisciplinary rehabilitation were the same as those included in the subgroup analysis on the effects on ADL. Since long-term multidisciplinary rehabilitation is beneficial for ADL, such intervention might reduce the need to increase the antiparkinsonian medication dose.

The quality of evidence measured by the GRADE approach ranged from very low to low by downgrading (1) the risk of bias due to the blinding of participants and personnel, (2) inconsistency, 
(3) imprecision due to the small sample size and wide confidence intervals, (4) indirectness, and (5) publication bias. Blinding participants and blinding personnel are common limitations in physiotherapy trials where the intervener and the patient perform face-to-face interventions and evaluations. However, as described in a previous study of remotely supervised aerobic exercise [19], the risk of bias in relation to the blinding of personnel could be mitigated by the participants themselves intervening remotely via a web-based system with a motivational app.

Several study limitations and future studies should be discussed. First, this study entails a wide range of interventions, but the number of studies of each intervention type was relatively small. Therefore, it was not possible to determine the difference in the effects of different types of interventions. During the review process, there were several studies that could not be included in this review because the medication state of evaluated patients or the means and SDs of change values in outcomes were not clear. Further wellreported quality RCTs will be needed to establish evidence of the most beneficial types of physiotherapy intervention in mild-to-moderate PD patients. In addition, we included both no-intervention and control-intervention groups together as controls. In 4 of the 10 studies, the control group was set as the group provided with a mild control intervention (e.g., stretching and relaxation, non-progressive exercise) $[10,14,15,19]$. Although these interventions were mild, their impact on the results cannot be completely ruled out. In the future, meta-analyses including sufficient numbers of quality studies with and without control intervention will be needed, so that they can be analyzed separately.

This review provided evidence that long-term physiotherapy interventions for 6 months or longer have a beneficial impact on motor symptoms in off medication state and antiparkinsonian medication dose in mild-to-moderate PD patients. As the disease progresses, PD patients still experience off states when they are taking antiparkinsonian medication, so the evidence that long-term physiotherapy improves motor symptoms in the off medication state is significant for PD patients. These results could convey the significance of long-term physiotherapy to clinicians and PD patients and motivate to implement long-term physiotherapy from the early to mid-stage of disease. Since only evaluating motor symptoms in on medication state may mask the effects of long-term physiotherapy, it is necessary to evaluate motor symptoms in off medication state as well, and to present the effects to PD patients in order to motivate them. For this purpose, it may be useful to use a telemedicine system that can assess motor symptoms even in off medication state [32]. This would make PD patients more aware of the benefits of long-term physiotherapy, which in turn could result in better adherence. If long-term physiotherapy reduces the antiparkinsonian medication dose, it may also reduce the risk of motor complications (such as motor fluctuations and dyskinesia) associated with the increases in medication dose over the disease course, and the healthcare costs associated with the rapid increase in the number of PD patients worldwide [33].

\section{ACKNOWLEDGMENTS}

We thank Mr. Koki Kishida, Mr. Haruka Komatsu, Miss Kanako Sakuta, and Risa Sugimori for objectively discussing the screening and analysis together. This work was supported by JSPS KAKENHI Grant Number 17K01545.

\section{CONFLICT OF INTEREST}

The authors have no financial conflict of interest related to this study.

\section{SUPPLEMENTARY MATERIAL}

The supplementary material is available in the electronic version of this article: https://dx.doi.org/ 10.3233/JPD-212782.

\section{REFERENCES}

[1] Reinoso G, Allen JC Jr, Au WL, Seah SH, Tay KY, Tan LC (2015) Clinical evolution of Parkinson's disease and prognostic factors affecting motor progression: 9-year follow-up study. Eur J Neurol 22, 457-463.

[2] Shulman LM, Gruber-Baldini AL, Anderson KE, Vaughan CG, Reich SG, Fishman PS, Weiner WJ (2008) The evolution of disability in Parkinson disease. Mov Disord 23, 790-796.

[3] Hariz GM, Forsgren L (2011) Activities of daily living and quality of life in persons with newly diagnosed Parkinson's disease according to subtype of disease, and in comparison to healthy controls. Acta Neurol Scand 123, 20-27.

[4] Ahlskog JE, Uitti RJ (2010) Rasagiline, Parkinson neuroprotection, and delayed-start trials: Still no satisfaction? Neurology 74, 1143-1148.

[5] Mizuno Y, Shimoda S, Origasa H (2018) Long-term treatment of Parkinson's disease with levodopa and other adjunctive drugs. J Neural Transm (Vienna) 125, 35-43.

[6] Schrag A, Quinn N (2000) Dyskinesias and motor fluctuations in Parkinson's disease. A community-based study. Brain 123, 2297-2305. 
[7] Armstrong MJ, Okun MS (2020) Diagnosis and treatment of Parkinson disease: A review. JAMA 323, 548-560.

[8] Tomlinson CL, Patel S, Meek C, Clarke CE, Stowe R, Shah L, Sackley CM, Deane KH, Herd CP, Wheatley K, Ives N (2012) Physiotherapy versus placebo or no intervention in Parkinson's disease. Cochrane Database Syst Rev (7), CD002817.

[9] Radder DLM, Lígia Silva de Lima A, Domingos J, Keus SHJ, van Nimwegen M, Bloem BR, de Vries NM (2020) Physiotherapy in Parkinson's disease: A meta-analysis of present treatment modalities. Neurorehabil Neural Repair 34, 871-880.

[10] Corcos DM, Robichaud JA, David FJ, Leurgans SE, Vaillancourt DE, Poon C, Rafferty MR, Kohrt WM, Comella CL (2013) A two-year randomized controlled trial of progressive resistance exercise for Parkinson's disease. Mov Disord 28, 1230-1240.

[11] Ferreira RM, Alves WMGDC, de Lima TA, Alves TGG, Alves Filho PAM, Pimentel CP, Sousa EC, Cortinhas-Alves EA (2018) The effect of resistance training on the anxiety symptoms and quality of life in elderly people with Parkinson's disease: A randomized controlled trial. Arq Neuropsiquiatr 76, 499-506.

[12] Frazzitta G, Bertotti G, Riboldazzi G, Turla M, Uccellini D, Boveri N, Guaglio G, Perini M, Comi C, Balbi P, Maestri $R$ (2012) Effectiveness of intensive inpatient rehabilitation treatment on disease progression in parkinsonian patients: A randomized controlled trial with 1-year follow-up. $\mathrm{Neu}$ rorehabil Neural Repair 26, 144-150.

[13] Frazzitta G, Maestri R, Bertotti G, Riboldazzi G, Boveri N, Perini M, Uccellini D, Turla M, Comi C, Pezzoli G, Ghilardi MF (2015) Intensive rehabilitation treatment in early Parkinson's disease: A randomized pilot study with a 2-year follow-up. Neurorehabil Neural Repair 29, 123-131.

[14] Nadeau A, Pourcher E, Corbeil P (2014) Effects of 24 wk of treadmill training on gait performance in Parkinson's disease. Med Sci Sports Exerc 46, 645-655.

[15] Schenkman M, Hall DA, Barón AE, Schwartz RS, Mettler P, Kohrt WM (2012) Exercise for people in early- or mid-stage Parkinson disease: A 16-month randomized controlled trial. Phys Ther 92, 1395-1410.

[16] Schenkman M, Moore CG, Kohrt WM, Hall DA, Delitto A, Comella CL, Josbeno DA, Christiansen CL, Berman BD, Kluger BM, Melanson EL, Jain S, Robichaud JA, Poon C, Corcos DM (2018) Effect of high-intensity treadmill exercise on motor symptoms in patients with de novo Parkinson disease: A phase 2 randomized clinical trial. JAMA Neurol 75, 219-226.

[17] Tollár J, Nagy F, Kovács N, Hortobágyi T (2019) Twoyear agility maintenance training slows the progression of parkinsonian symptoms. Med Sci Sports Exerc 51, 237-245.

[18] van der Kolk NM, de Vries NM, Penko AL, van der Vlugt M, Mulder AA, Post B, Alberts JL, Bloem BR (2018) A remotely supervised home-based aerobic exercise programme is feasible for patients with Parkinson's disease: Results of a small randomised feasibility trial. J Neurol Neurosurg Psychiatry 89, 1003-1005.

[19] van der Kolk NM, de Vries NM, Kessels RPC, Joosten H, Zwinderman AH, Post B, Bloem BR (2019) Effectiveness of home-based and remotely supervised aerobic exercise in Parkinson's disease: A double-blind, randomised controlled trial. Lancet Neurol 18, 998-1008.
[20] Mak MK, Wong-Yu IS, Shen X, Chung CL (2017) Longterm effects of exercise and physical therapy in people with Parkinson disease. Nat Rev Neurol 13, 689-703.

[21] Keus SH, Munneke M, Nijkrake MJ, Kwakkel G, Bloem BR (2009) Physical therapy in Parkinson's disease: Evolution and future challenges. Mov Disord 24, 1-14.

[22] Keus SH, Munneke M, Graziano M, Paltamaa J, Pelosin E, Domingos J, Brühlmann S, Ramaswamy B, Prins J, Struiksma C, Rochester L, Nieuwboer A, Bloem BR (2014) European Physiotherapy Guideline for Parkinson's Disease. KNGF/ParkinsonNet, the Netherlands.

[23] Guyatt GH, Oxman AD, Vist GE, Kunz R, Falck-Ytter Y, Alonso-Coello P, Schünemann HJ; GRADE Working Group (2008) GRADE: An emerging consensus on rating quality of evidence and strength of recommendations. BMJ 336, 924-926.

[24] Movement Disorder Society Task Force on Rating Scales for Parkinson's Disease (2003) The Unified Parkinson's Disease Rating Scale (UPDRS): Status and recommendations. Mov Disord 18, 738-750.

[25] Goetz CG, Tilley BC, Shaftman SR, Stebbins GT, Fahn S, Martinez-Martin P, Poewe W, Sampaio C, Stern MB, Dodel R, Dubois B, Holloway R, Jankovic J, Kulisevsky J, Lang AE, Lees A, Leurgans S, LeWitt PA, Nyenhuis D, Olanow CW, Rascol O, Schrag A, Teresi JA, van Hilten JJ, LaPelle N; Movement Disorder Society UPDRS Revision Task Force (2008) Movement Disorder Society-sponsored revision of the Unified Parkinson's Disease Rating Scale (MDS-UPDRS): Scale presentation and clinimetric testing results. Mov Disord 23, 2129-2170.

[26] Tomlinson CL, Stowe R, Patel S, Rick C, Gray R, Clarke CE (2010) Systematic review of levodopa dose equivalency reporting in Parkinson's disease. Mov Disord 25, 26492653.

[27] Higgins JP, Thomas J, Chandler J, Cumpston M, Li T, Page MJ, Welch VA (2019) Cochrane handbook for systematic reviews of interventions, 2nd Edition, John Wiley \& Sons, Chichester (UK).

[28] Liberati A, Altman DG, Tetzlaff J, Mulrow C, Gøtzsche PC, Ioannidis JP, Clarke M, Devereaux PJ, Kleijnen J, Moher D (2009) The PRISMA statement for reporting systematic reviews and meta-analyses of studies that evaluate healthcare interventions: Explanation and elaboration. BMJ 339, b2700.

[29] Schootemeijer S, van der Kolk NM, Bloem BR, de Vries NM (2020) Current perspectives on aerobic exercise in people with Parkinson's disease. Neurotherapeutics 17, 1418-1433.

[30] Petzinger GM, Fisher BE, McEwen S, Beeler JA, Walsh JP, Jakowec MW (2013) Exercise-enhanced neuroplasticity targeting motor and cognitive circuitry in Parkinson's disease. Lancet Neurol 12, 716-726.

[31] Sturkenboom IH, Graff MJ, Hendriks JC, Veenhuizen Y, Munneke M, Bloem BR, Nijhuis-van der Sanden MW; OTiP study group (2014) Efficacy of occupational therapy for patients with Parkinson's disease: A randomised controlled trial. Lancet Neurol 13, 557-566.

[32] Sekimoto S, Oyama G, Chiba S, Nuermaimaiti M, Sasaki F, Hattori N (2020) Holomedicine: Proof of the concept of interactive three-dimensional telemedicine. Mov Disord 35, 1719-1720.

[33] Dorsey ER, Bloem BR (2018) The Parkinson pandemic-a call to action. JAMA Neurol 75, 9-10. 